\title{
Photonics Society of Poland: towards a new decade
}

\author{
Tomasz R. Woliński \\ Photonics Society of Poland President
}

ReceivedJune 29, 2017; published June 30, 2017

Photonics Society of Poland (PSP) was transformed from the SPIE Poland Chapter on October 18, 2007 and almost 10 years after with its 235 PSP members (as of 8.06.2017) - including 22 SPIE members, 12 SPIE Fellows, and 2 OSA Fellows - is still the largest optics and photonics organization in Poland. On March 9, 2017 a new PSP Board and Review Committee for the term 2017-2021 have been elected, please refer to http://photonics.pl.

Annually or bi-annually,PSP co-organizers several national and international conferences in Poland as Szczyrk Conferences on Integrated Optics: Sensors, Sensing Structures and Methods,IOS http://ios.polsl.pl/; WILGA Symposia on Photonics and Web Engineering: http://wilga.ise.pw.edu.pl/; Conferences of Liquid Crystals Chemistry, Physics and Applications,http://www.clc16.plconferences on Optical Fibers and Their Applications OFTA, http://we.pb.edu.pl/swiatlowody/

Photonics Society of Poland is an active member of the Polish Technological Platform on Photonics (www.pptf.pl) established in 2013 and supports also 6 joint SPIE/OSA Students Chapters in Warsaw, Wrocław, Lublin, Gliwice, Toruń, and Gdańsk. In 2017, PSP has renewed its long-term collaboration agreements with the leading international optics and photonics societies: SPIE and OSA.

Since 2009, Photonics Society of Poland has been editing (in collaboration with SPIE) Photonics Letters of Poland (PLP). The current, $34^{\text {th }}$ PLP issue contains 7 papers related with the European COST Action IC1208 Integrating Devices and Materials and 6 regular papers. The diversity of the work reported here covers a large spectrum of technology, theory and characterization of photonic material, systems, and devices.

Laudyn et al. discusses light localization in nematic liquid crystals that supports vector nematicons.Nersesyan et al. reports on the switching of a planar nematic liquid crystal cell by means of electric voltage applied and with assistance of optical field. In particular, several switching conditions are demonstrated while obtained for different tilt angles of incident light beams. In letter by Benito et al. the light interaction between the incoming and scattered electric fields in dimers made of different semiconducting materials is explored. Authors prove that interferential effects obtained by directing the scattered light towards the gap of the dimer give rise to creation of all-optical nanoswitchers. Plasmonic coupling induced by growing processes of metal nanoparticles in wrinkled surface of a large area PDMS template is presented by $\mathrm{U}$. Cataldi and T. Buergi. Studies on electrical tuning of optical channels formed by the PDMS waveguide channels infiltrated with liquid crystalline material can be found in a letter by Rutkowska et al. Apart to numerical simulations demonstrating changes in optical parameters of the waveguide channels when influenced by electric field, first experimental trials on sputtering the ITO layer on PDMS are also presented. Budaszewski et al. investigates light propagation in a photonic crystal fiber infiltrated with mesogenic azobenzene dyes. Presented results may lead to optically tunable photonic devices of a new design. Finally, in the last paper related to the COST Action and written by Orzechowski et al.a new alternative method for the refractive index measurement in blue phase liquid crystal basing on the Kerr effect is presented.

Ojeda-Castañedaet al. discloses the use of a suitable pair of phase-only masks for generating either sinusoidal phase gratings or sinusoidal phase zone plates with tunable optical path delays. Compression of computergenerated holograms in image projection is presented by Kochańska and Makowski who elaborated efficient algorithms of lossless compression to transfer calculations to the cloud.Amraouiet al.Present preparationmethods of silver nanospheroids of different sizes and forms while Hasan et al.numerically demonstrate a hybrid-core microstructure fiber for ow-loss terahertz guidance. Grigorian and Cenian discuss an influence of nitrogen on plasma discharge of $\mathrm{CO}$ lasers in plasma-chemical processes.Finally, Razak et al.demonstrated a passively Q-switched Erbium-doped fiber laser by using a black phosphorus saturable absorber as the Q-switcher. 